BMJ Open Diabetes

Research \& Care

\title{
Urinary adiponectin as a new diagnostic index for chronic kidney disease due to diabetic nephropathy
}

Shinnosuke Yamakado, ${ }^{1}$ Hiroki Cho, ${ }^{1}$ Mikio Inada, ${ }^{1}$ Mika Morikawa, ${ }^{2}$ Yong-Huang Jiang, ${ }^{2}$ Kenji Saito, ${ }^{2}$ Kazunari Nakaishi, ${ }^{2}$ Satoshi Watabe, ${ }^{2}$ Hitomi Takagi, ${ }^{3}$ Mugiho Kaneda, ${ }^{3}$ Akira Nakatsuma, ${ }^{3}$ Masaki Ninomiya, ${ }^{3}$ Hitomi Imachi, ${ }^{4}$ Takeshi Arai, ${ }^{4}$ Takuo Yoshimoto, ${ }^{4}$ Koji Murao, ${ }^{4}$ Jyun-Hao Chang, ${ }^{5}$ Shih-Min Chen, ${ }^{5}$ Yi-Chen Shih, ${ }^{5}$ Min-Jing Zeng, ${ }^{5}$ Liang-Yin Ke, ${ }^{\circ}$ Chu-Huang Chen, ${ }^{\circ}$ Teruki Yoshimura, ${ }^{6}$ Toshiaki Miura, ${ }^{7}$ Etsuro Ito ${ }^{\oplus 1,8}$

To cite: Yamakado S, Cho $\mathrm{H}$, Inada $\mathrm{M}$, et al. Urinary adiponectin as a new diagnostic index for chronic kidney disease due to diabetic nephropathy. BMJ Open Diab Res Care 2019;7:e000661. doi:10.1136/ bmjdrc-2019-000661

- Additional material is published online only. To view please visit the journal online (http://dx.doi.org/10. 1136bmjdrc-2019-000661).

SY and $\mathrm{HC}$ contributed equally.

Received 12 February 2019 Revised 18 April 2019 Accepted 3 May 2019
Check for updates

\section{(C) Author(s) (or their} employer(s)) 2019. Re-use permitted under CC BY-NC. No commercial re-use. See rights and permissions. Published by BMJ.

For numbered affiliations see end of article.

Correspondence to Prof Etsuro Ito; eito@waseda.jp

\section{ABSTRACT}

Objective The chronic kidney disease (CKD) is widely diagnosed on the basis of albuminuria and the glomerular filtration rate. A more precise diagnosis of $\mathrm{CKD}$, however, requires the assessment of other factors. Urinary adiponectin recently attracted attention for CKD assessment, but evaluation is difficult due to the very low concentration of urinary adiponectin in normal subjects. Research design and methods We developed an ultrasensitive ELISA coupled with thionicotinamide-adenine dinucleotide cycling to detect trace amounts of proteins, which allows us to measure urinary adiponectin at the subattomole level. We measured urinary adiponectin levels in 59 patients with diabetes mellitus (DM) and 24 subjects without DM (normal) to test our hypothesis that urinary adiponectin levels increase with progression of CKD due to DM.

Results The urinary adiponectin levels were 14.88 \pm 3.16 $(\mathrm{ng} / \mathrm{mg}$ creatinine, mean \pm SEM) for patients with DM, and $3.06 \pm 0.33$ ( $\mathrm{ng} / \mathrm{mg}$ creatinine) for normal subjects. The threshold between them was $4.0 \mathrm{ng} / \mathrm{mg}$ creatinine. The urinary adiponectin levels increased with an increase in the CKD risk. Furthermore, urinary adiponectin mainly formed a medium-molecular weight multimer (a hexamer) in patients with DM, whereas it formed only a lowmolecular weight multimer (a trimer) in normal subjects. That is, the increase in urinary adiponectin in patients with DM led to the emergence of a medium-molecular weight form in urine.

Conclusions Our new assay showed that urinary adiponectin could be a new diagnostic index for CKD. This assay is a non-invasive test using only urine, thus reducing the patient burden.

\section{INTRODUCTION}

Chronic kidney disease (CKD) is mainly caused by diabetes mellitus (DM) and hypertension, which alter the function and structure of the kidney over the long term. ${ }^{1}$ According to international guidelines, CKD is defined as meeting at least one of the following two criteria for at least 3 months $^{2-4}$ : (A) estimated glomerular filtration rate (eGFR) less than

\section{Significance of this study}

What is already known about this subject?

- Our preliminary results of the measurement of urinary adiponectin with an ELISA that we developed suggest that the urinary adiponectin levels are higher in patients with diabetes mellitus (DM) than in normal (non-DM) subjects.

- We thus hypothesized that urinary adiponectin levels tend to increase with an increase in the risk of diabetic nephropathy.

What are the new findings?

- The urinary adiponectin levels increased with an increase in the chronic kidney disease (CKD) risk.

- Urinary adiponectin mainly formed a medium-molecular weight multimer in patients with DM, whereas it formed only a low-molecular weight multimer in normal subjects.

How might these results change the focus of research or clinical practice?

- Urinary adiponectin can be a new diagnostic index for CKD.

- Our assay is a non-invasive test using only urine, thus reducing the patient burden.

$60 \mathrm{~mL} / \mathrm{min}$ per $1.73 \mathrm{~m}^{2}$, and (B) presence of marker (one or more) of kidney damage, for example, albuminuria (albumin-to-creatinine ratio $(A C R) \geq 30 \mathrm{mg} / \mathrm{g}$ ). This CKD classification system offers the advantage that the categories determined by this system reflect a patient's risk of multiple adverse outcomes, and it is therefore a useful and simple tool for determining risk stratification in clinical practice. ${ }^{5}$ The Work Group in the National Kidney Foundation in the USA, however, has pointed out a number of limitations to the current definition and classification of $\mathrm{CKD}^{6}$; the known markers of kidney damage are not 
sensitive and the association of the eGFR with complications of CKD is not necessarily causal. A new diagnostic index for CKD is long overdue.

Adiponectin is an adipocyte-derived vasoactive peptide with anti-inflammatory and insulin-sensitizing peptides, as well as cardioprotective effects on endothelial cells, and is increased in obesity and DM. ${ }^{78}$ Serum adiponectin is believed to enhance insulin sensitivity, and individuals with obesity, type $2 \mathrm{DM}$, and other metabolic disorders often have low serum adiponectin levels. ${ }^{9}{ }^{10}$ In contrast to the serum levels, our preliminary results of the measurement of urinary adiponectin with an ultrasensitive ELISA that we developed suggest that the urinary adiponectin levels are higher in patients with DM than in normal (non-DM) subjects. ${ }^{11}$ We thus hypothesized that urinary adiponectin levels tend to increase with an increase in the risk of diabetic nephropathy.

Recent studies demonstrated the validity of urinary adiponectin as a marker of the progression of focal segmental glomerulosclerosis, ${ }^{12}$ albuminuria, ${ }^{13}$ incident cardiovascular disease, and renal function deterioration, ${ }^{14}$ as well as diabetic nephropathy. ${ }^{15-19}$ The sensitivity of commercially available ELISA kits, ${ }^{16}{ }^{17}$ however, is insufficient for the measurement of the low level of urinary adiponectin in normal subjects, ${ }^{20}$ and radioimmunoassay ${ }^{15}$ and more complicated ELISAs ${ }^{19}$ are not practical for application in most clinics and hospitals. Using our ultrasensitive ELISA, however, which only requires an ordinary microplate reader and no special apparatus, we could sufficiently detect the low urinary adiponectin levels in normal subjects. ${ }^{11}$ More recently, some research groups have also noticed the relationship between adiponectin levels and diabetic nephropathy. ${ }^{21}$ In particular, Christou and Kiortsis have discussed the renal elimination of adiponectin. ${ }^{22}$

In the present study, we measured the urinary adiponectin levels in 59 patients with DM and 24 normal subjects with our ultrasensitive ELISA, and compared the data with the conventional CKD classification using eGFR and ACR. Our results suggest that urinary adiponectin could be used as a new diagnostic index for CKD.

\section{RESEARCH DESIGN AND METHODS \\ Sample collection}

All the authors ensure that the planning, conduct, and reporting of human research are in accordance with the Helsinki Declaration as revised in 2013. The present study was approved by the ethics committees at Waseda University, Tokushima Bunri University, and Kagawa University. The studies dealing with human samples were performed at these three universities, and thus the authors from the other universities visited these three universities for the experiments, or analyzed the data and wrote the manuscript at their own universities. Urine was collected from 59 patients with DM and 24 normal subjects (ie, subjects that did not have DM or hypertension). The mean age for 59 patients with $\mathrm{DM}$ was $68.6 \pm 1.4($ mean \pm SEM) , and that for 24 normal subjects was $33.3 \pm 2.2$. Sera were collected from 58 patients with DM and six normal subjects (see online supplementary tables 1 and 2). The mean age for 58 patients with DM was $68.5 \pm 1.4$, and that for six normal subjects was $24.0 \pm 0.9$. The normal subjects were confirmed not to have DM or hypertension based on a medical examination conducted at their workplace. All the samples were collected in the Kagawa area in Japan. Table 1 shows the conventional CKD classification using the eGFR and albuminuria (ie, ACR) for the patients with DM. In addition, urine samples used for western blotting to test the effects of exercise were collected from three normal subjects in the Tokyo area independent of the above-mentioned Kagawa area subjects.

\section{Ultrasensitive ELISA for detection of urinary and serum adiponectin}

An ultrasensitive ELISA coupled with thionicotinamide-adenine dinucleotide (thio-NAD) cycling was originally developed by Watabe and Ito. ${ }^{11}{ }^{23-26}$ Shortly, a sandwich method using a primary and a secondary

\begin{tabular}{|c|c|c|c|c|c|}
\hline & & ACR & A1 & A2 & A3 \\
\hline & & (mg/g Cr) & $<30$ & $30-299$ & $\geq 300$ \\
\hline \multirow{6}{*}{$\begin{array}{l}\text { eGFR } \\
\left(\mathrm{mL} / \mathrm{min} / 1.73 \mathrm{~m}^{2}\right)\end{array}$} & G1 & $\geq 90$ & $5,22,28,30,59$ & & \\
\hline & G2 & $60-89$ & $\begin{array}{l}1,6,10,11,16,18,25 \\
27,29,44,47,49\end{array}$ & $8,15,57$ & $9,14,43,50,55$ \\
\hline & G3a & $45-59$ & $7,19,21,31,32,46$ & $2,17,20,42$ & $\begin{array}{l}12,13,24,26 \\
38,45,52,54\end{array}$ \\
\hline & G3b & $30-44$ & 3,48 & 51 & 56 \\
\hline & G4 & $15-29$ & $4,36,39,40$ & $23,34,53,58$ & 33 \\
\hline & G5 & $<15$ & $35,37,41$ & & \\
\hline
\end{tabular}

Numbers in this table are the DM patient numbers. Shaded areas indicate the risk according to CKD classification. The darker the shading, the higher the CKD classification.

ACR, albumin-to-creatinine ratio; CKD, chronic kidney disease; DM, diabetes mellitus; eGFR, estimated glomerular filtration rate. 
antibody for antigens is employed in an ELISA. An androsterone derivative, $3 \alpha$-hydroxysteroid, is produced by the hydrolysis of $3 \alpha$-hydroxysteroid 3-phosphate with alkaline phosphatase (ALP, EC 3.1.3.1) linked to the secondary antibody. This $3 \alpha$-hydroxysteroid is oxidized to a 3-ketosteroid by $3 \alpha$-hydroxysteroid dehydrogenase (3 $\alpha$-HSD, EC 1.1.1.50) with a cofactor thio-NAD. By the opposite reaction, the 3-ketosteroid is reduced to a $3 \alpha-$-hydroxysteroid by $3 \alpha-H S D$ with a cofactor NADH. During this cycling reaction, thio-NADH accumulates in a triangular number fashion. Accumulated thio-NADH can be measured directly at an absorbance of $405 \mathrm{~nm}$ without any interference from other cofactors. ${ }^{23}$

The measurement methods for adiponectin were slightly modified from the previous studies. ${ }^{11}$ The antigen used was a recombinant human adiponectin derived from a human adiponectin oligomers/Acrp30 quantitative ELISA kit (Cat No DHWAD0; R\&D Systems, Minneapolis, MN, USA). The ELISA part is a sandwich ELISA in which the primary and secondary antibodies for anti-adiponectin were the human adiponectin/ Acrp30 monoclonal antibodies (Cat No MAB10651500 and MAB1065-500, respectively; R\&D Systems). The second antibody was linked with ALP (origin from calf intestine, recombinant by Pichia pastoris; Cat No 03359123001; Roche, Mannheim, Germany). As the primary substrate, we used $17 \beta$-methoxy-5 $\beta$-androstan-3 $\alpha$-ol 3-phosphate synthetized from $5 \beta$-androsterone. ${ }^{23}$ In the thio-NAD cycling part, $3 \alpha$-HSD kindly gifted from TAUNS (Izunokuni, Shizuoka, Japan) was used. Thio-NAD was purchased from Oriental Yeast (Cat No 44101900; Tokyo, Japan) and NADH was purchased from Roche.

A $100 \mu \mathrm{L}$ solution of primary antibody, which was adjusted to $2 \mu \mathrm{g} / \mathrm{mL}$ in $50 \mathrm{mM} \mathrm{Na}_{2} \mathrm{CO}_{3}$ (pH 9.6), was added to wells of microplates and incubated for 1 hour at room temperature. The microplates were incubated with $1 \%$ bovine serum albumin (BSA) in Tris-buffered saline (TBS) for 1 hour at room temperature. The 100 $\mu \mathrm{L}$ sample of urine or serum was added to each well and incubated for 1 hour at $37^{\circ} \mathrm{C}$. Here, the samples of urine and serum were diluted with TBS including $0.1 \%$ BSA. This dilution was taken account when approximating the sensitivity according to the results of the spike-andrecovery tests (see online supplementary data). Then, a $100 \mu \mathrm{L}$ solution of secondary antibody, which was conjugated with ALP and adjusted to $10 \mathrm{pmol} / \mathrm{mL}$ in TBS including $0.05 \%$ Tween $20 \%$ and $0.1 \%$ BSA, was added to the wells. To amplify the ELISA signals, a $100 \mu \mathrm{L}$ solution of thio-NAD cycling was added to the wells. This solution contained $1.0 \mathrm{mM}$ NADH, $2.0 \mathrm{mM}$ thio-NAD, $0.15 \mathrm{mM}$ $17 \beta$-methoxy-5 $\beta$-androstan-3 $\alpha$-ol 3-phosphate, and $10 \mathrm{U} /$ $\mathrm{mL} 3 \alpha-\mathrm{HSD}$ in $100 \mathrm{mM}$ Tris-HCl (pH 9.5). The detectable signal (ie, thio-NADH) accumulated in a triangulated manner during the cycling reaction within a short measurement time, and was measured with a grating microplate reader at $405 \mathrm{~nm}$.

\section{Western blotting}

To analyze multimers of urinary adiponectin, we first concentrated the adiponectin in urine. Ammonium sulfate was added to the urine sample to a concentration of $50 \%$. The sample was centrifuged at $25000 \times g$ for 30 $\min$ at $4^{\circ} \mathrm{C}$, and the precipitate was diluted with $3 \mathrm{~mL}$ of $1 \times$ TBS. This diluted precipitation was placed in a dialysis bag and dialyzed in more than $75 \mathrm{~mL}$ of $1 \times$ TBS. The sample was then incubated in the buffer overnight at $4^{\circ} \mathrm{C}$. After centrifugation at $2000 \mathrm{rpm}(330 \times \mathrm{g})$ for $3 \mathrm{~min}$, the supernatant was collected. This collected sample, which was again placed in a dialysis bag, was set in a glass bottle with a negative pressure of $93 \mathrm{kPa}(=70 \mathrm{cmHg})$ and kept overnight at $4^{\circ} \mathrm{C}$. The sample was diluted with a $2 \mathrm{~mL}$ $1 \times$ TBS.

Sodium dodecyl sulfate-polyacrylamide gel electrophoresis was performed using a commercial gel (Mini-PROTEAN TGM precast gels, Bio-Rad Laboratories, Hercules, CA, USA). The membrane transfer was performed using a semidry system. The blotted membrane was blocked with $1 \%$ BSA solution, and the membrane was probed with a primary antibody. We used antiadiponectin antibody of clone 166126 (R\&D Systems), except in the exercise experiments. For the exercise experiments, we used two different antiadiponectin antibodies: clone 166128 (R\&D Systems), which was the same as the secondary antibody for the ultrasensitive ELISA; and antiadiponectin antibody [19F1] ab22554 (Abcam, Cambridge, UK). These antibodies were applied to the membrane at a 1:1000 dilution. The membrane was incubated with one of these antibodies for 1 hour at room temperature. The membrane was incubated with the secondary antibody (horseradish peroxidase-conjugated anti-mouse antibody, Dako, Agilent Technologies, Santa Clara, CA, USA) at a 1:4000 dilution for 1 hour at room temperature. Then, the membrane was incubated with a DAB Substrate Kit (SK-4100, Vector Laboratories, Burlingame, CA, USA) for $10 \mathrm{~min}$ at room temperature. The membrane was viewed with a chemiluminescence detection apparatus.

\section{Other biochemical measurements}

Urinary creatinine was measured using a creatinine assay kit (K625-100; BioVision, Milpitas, CA, USA). Other biochemical data were analyzed in the Clinical Laboratory in Kagawa University Hospital and in the Laboratory of Medical Pharmacy in Kagawa School of Pharmaceutical Sciences, Tokushima Bunri University.

\section{Exercise}

To examine changes in the amount and type of multimers of urinary adiponectin induced by exercise, urine samples were collected from three normal subjects before and after an anaerobic exercise and an aerobic exercise. The anaerobic exercise included bench press exercise ( 3 sets of 6 repetitions), crunch exercise (3 sets of 30 repetitions), chinning exercise ( 3 sets of 6 repetitions), arm curl exercise ( 3 sets of 6 repetitions), and leg press exercise ( 3 sets 
of 6 repetitions). The total time for the anaerobic exercise was 1 hour. The aerobic exercise was jogging at $6 \mathrm{~km} /$ hour for $30 \mathrm{~min}$. The urine samples were used to evaluate both the amount of change by the ultrasensitive ELISA and the multimer change by western blotting.

\section{Statistical analyses}

Data are expressed as mean \pm SEM. Significant differences at $\mathrm{p}<0.05$ were evaluated by paired t-test, Mann-Whitney $\mathrm{U}$ test, two-way analysis of variance, or the Kruskal-Wallis test as appropriate. We also used the Tukey test or Scheffé test for multiple comparisons. The limit of detection was estimated from the mean of the blank, the SD of the blank, and a confidence factor of 3 . The limit of quantification was estimated by the same method as used for the limit of detection, but with a confidence factor of 10 .

\section{RESULTS}

Calibration curves, limit of detection, limit of quantification, and coefficient of variation for adiponectin standard

When we used a human high-molecular weight (HMW) adiponectin (Cat No DHWAD0; R\&D Systems) as the reference, the ultrasensitive ELISA coupled with thio-NAD cycling in TBS yielded a linear calibration curve $\left(y=0.0046 x+0.016, R^{2}=0.99\right)$ in the range of $5-100$ $\mathrm{pg} / \mathrm{mL}$ (figure 1A). This curve was obtained from the absorbance of the accumulated thio-NADH at a cycling reaction time of $30 \mathrm{~min}$. The limit of detection was 0.81 $\mathrm{pg} / \mathrm{mL}$, corresponding to ca $2.7 \times 10^{-19}$ moles/assay, and the limit of quantification was $2.7 \mathrm{pg} / \mathrm{mL}$, corresponding to ca $9.0 \times 10^{-19}$ moles/assay. The molecular mass was assumed to be $300 \mathrm{kDa}$ and a single assay contained $100 \mu \mathrm{L}$ of solution. These values were apparently more sensitive than those of commercially available kits for human adiponectin (eg, Invitrogen kit: BMS2032-2 and BioVendor high sensitivity kit: RD191023100). This point is very important because urinary adiponectin levels in normal subjects are very low compared with those of patients with DM. ${ }^{11}$ To our knowledge, only our ultrasensitive ELISA, radioimmunoassay, and the other complicated ELISAs have been used to evaluate urinary adiponectin levels in normal subjects. ${ }^{11} 1519$ The coefficient of variation in our system was $2 \%$ for $0.1 \mathrm{ng} / \mathrm{mL}$ adiponectin standard.

\section{Adiponectin levels in urine and serum}

We measured concentrations of adiponectin and creatinine in urine collected from patients with DM and normal subjects, and expressed the urinary adiponectin levels relative to creatinine $(\mathrm{ng} / \mathrm{mg}$ creatinine; figure $1 \mathrm{~B}-\mathrm{D})$. The urinary adiponectin levels were $14.88 \pm 3.16(\mathrm{ng} / \mathrm{mg}$ creatinine, $\mathrm{n}=59$ ) for patients with $\mathrm{DM}$, and $3.06 \pm 0.33$ ( $\mathrm{ng} / \mathrm{mg}$ creatinine, $\mathrm{n}=24)$ for normal subjects. Thus, the urinary adiponectin levels were higher in patients with DM (Mann-Whitney U test, $\mathrm{p}<0.01$ ) than in normal subjects, and the threshold for discriminating between normal subjects and patients with DM, which was set as 4.0 $\mathrm{ng} / \mathrm{mg}$ creatinine in our previous study, ${ }^{11}$ was confirmed.
The serum adiponectin concentrations of patients with DM ranged from 1.29 to $22.30 \mu \mathrm{g} / \mathrm{mL}$ (mean $7.18 \mu \mathrm{g}$ / $\mathrm{mL}$ ) and those of normal subjects ranged from 4.91 to $10.04 \mu \mathrm{g} / \mathrm{mL}$ (mean $7.71 \mu \mathrm{g} / \mathrm{mL}$ ), and did not differ significantly between patients with DM and normal subjects (Mann-Whitney U test, $\mathrm{p}>0.05$; figure $1 \mathrm{E}, \mathrm{F}$ ).

\section{Relationship among urinary adiponectin level, conventional CKD classification, eGFR, and ACR}

According to the conventional CKD classification, 17 patients with DM in the present study were classified as normal; 9 patients were classified as low risk; 11 patients were classified as middle risk; and 22 patients were classified as high risk (table 1). The relationship between the urinary adiponectin levels and the conventional CKD classification showed that the urinary adiponectin level increased with progression of the CKD classification (Kruskal-Wallis test, $\mathrm{p}<0.01$, figure $2 \mathrm{~A}$ ). The urinary adiponectin level was not correlated with eGFR in patients with DM (figure 2B). The urinary adiponectin level was positively correlated with ACR in patients with DM $\left(R^{2}=0.85, \mathrm{p}<0.01\right.$, figure $\left.2 \mathrm{C}\right)$. The responsiveness of urinary adiponectin levels was much better than that of ACR, indicating the urinary adiponectin level is a reliable indicator of proteinuria. We found no correlation between eGFR and ACR in the patients with DM (figure 2D).

\section{Change in urinary adiponectin level by time or by exercise}

The samples used for the data shown in figures 1 and 2 were collected from patients with DM and normal subjects irrespective of the time of collection and the presence or absence of exercise before collection. To examine the first point, urine was collected from three normal subjects every 4 hours (eg, 08:00, 12:00, 16:00, and 20:00) in 1 day. We detected no significant difference in urinary adiponectin levels depending on the time of day the sample was collected (see online supplementary figure 1). On the other hand, we investigated whether urinary adiponectin levels change after exercise (figure 3A,B). Urine was collected from three normal subjects before and after performing anaerobic exercise and aerobic exercise. The urinary adiponectin levels were significantly higher after anaerobic exercise than before it $(\mathrm{n}=3$, paired t-test, $\mathrm{p}<0.05$, figure $3 \mathrm{~A})$, whereas there was no significant difference between before and after aerobic exercise $(n=3$, paired t-test, $p>0.05$, figure $3 B)$. Thus, even in normal subjects, urinary adiponectin levels exceed the threshold of $4.0 \mathrm{ng} / \mathrm{mg}$ creatinine after anaerobic exercise. Patients with DM, however, are not likely to have performed anaerobic exercise before going to the hospital.

\section{Western blotting to examine multimers}

In blood, adiponectin (monomer $=28 \mathrm{kDa}$ ) exists in three multimer forms: a trimer (low-molecular weight (LMW), molecular mass $84 \mathrm{kDa}$ ), a hexamer (trimer-dimer) of medium-molecular weight (MMW, $168 \mathrm{kDa}$ ), or a 
A

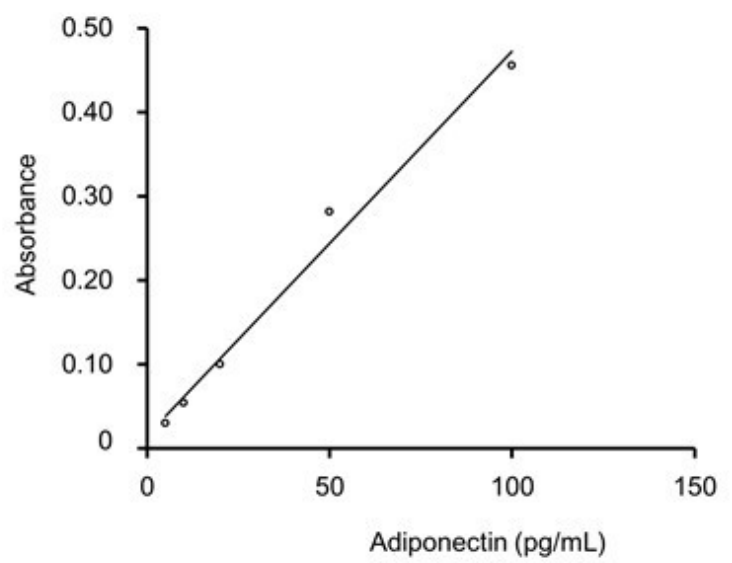

B

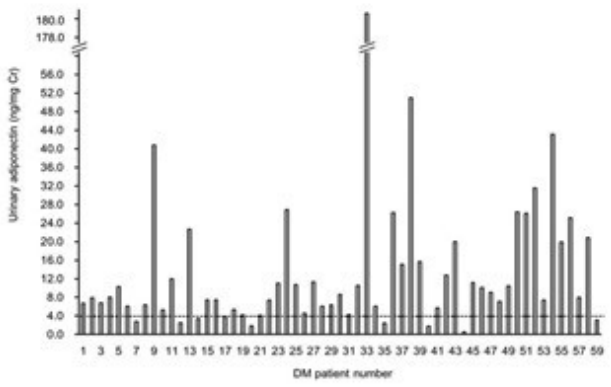

C

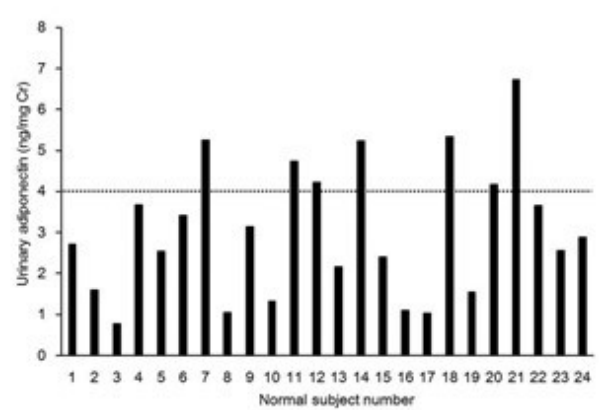
Normal subjoct number
$E$

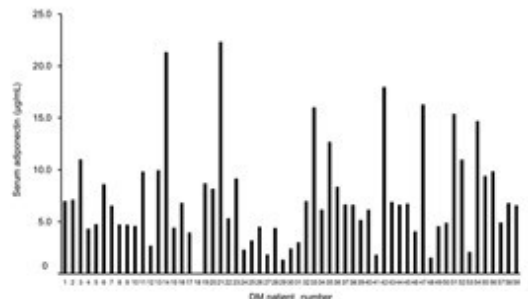

$\mathrm{F}$

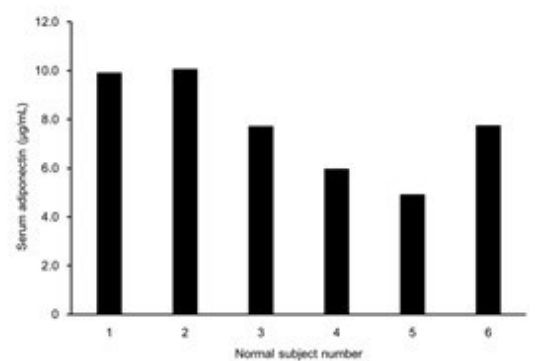
dinucleotide (thio-NAD) cycling. The blank value (ie, absorbance of $0 \mathrm{pg} / \mathrm{mL}$ adiponectin) was subtracted. The figure directly expresses the values corresponding to the adiponectin concentration. The linear calibration curve after a cycling reaction time of 30 min was $y=0.0046 x+0.016, R^{2}=0.99$ in the range of $5-100 \mathrm{pg} / \mathrm{mL}$. (B) Urinary adiponectin level for patients with DM. (C) Urinary adiponectin level in normal subjects. As determined on the basis of our previous study, the threshold adiponectin level distinguishing patients with DM from normal subjects is $4 \mathrm{ng} / \mathrm{mg}$ creatinine. (D) Merged graph of (B) and (C). The $x$-axis shows the urinary adiponectin levels (rounded up to the nearest integer). The $y$-axis shows the number of subjects. (E) Serum adiponectin level for patients with DM. (F) Serum adiponectin level for normal subjects. There was no significant difference between patients with DM and normal subjects. Some patients with DM had extremely high serum adiponectin levels. No sample was collected from patient No 18 with DM. 

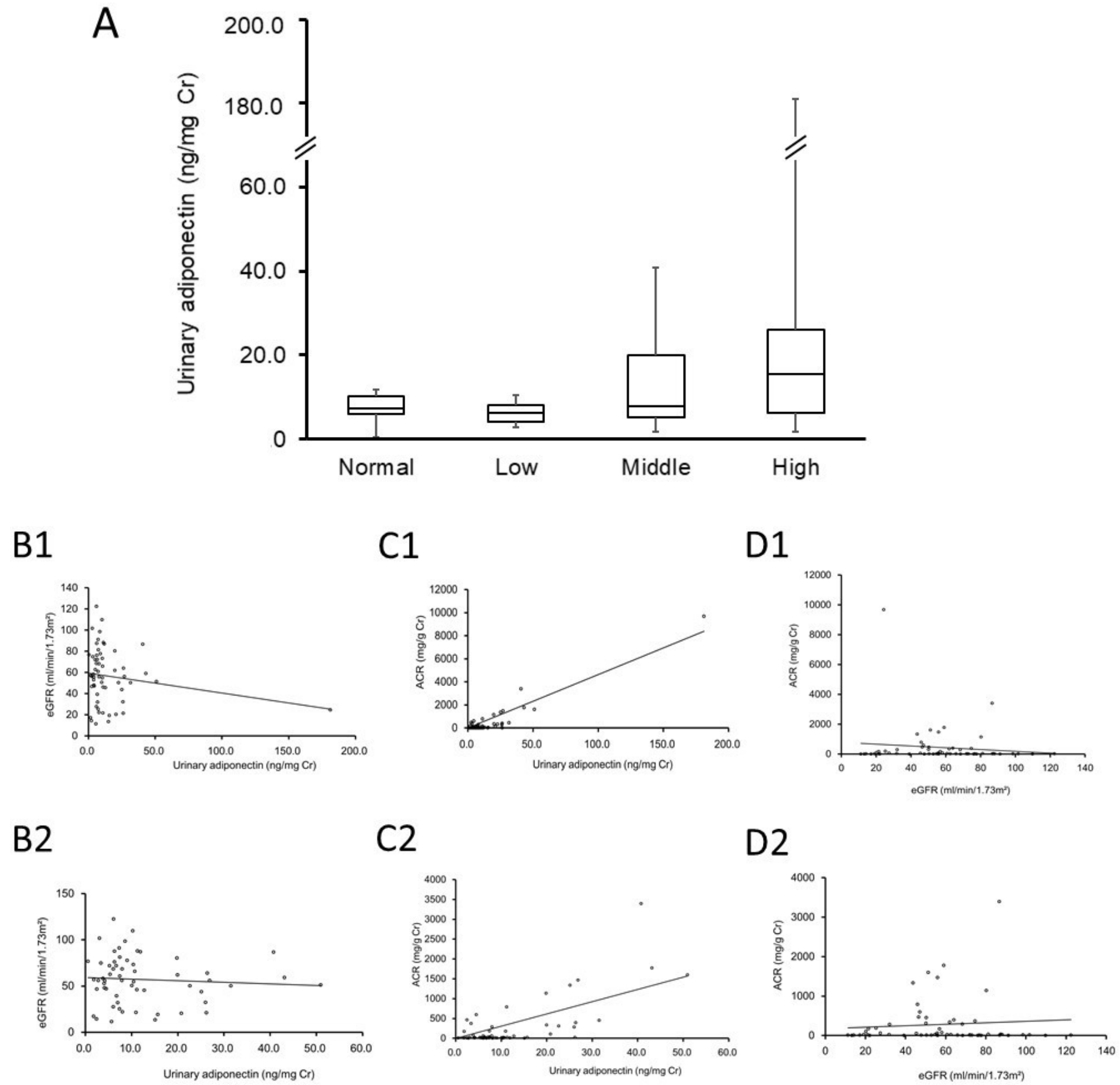

Figure 2 Correlations among chronic kidney disease (CKD) classification, urinary adiponectin level, eGFR, and ACR. (A) Correlation between CKD classification and urinary adiponectin level. The CKD classification has four stages for risk assessment: normal, low, middle, and high (see table 1). The data are expressed as box plots. The further the CKD progression, the higher the urinary adiponectin level. Kruskal-Wallis test, $p<0.01$. (B) Correlation between urinary adiponectin level and eGFR. (B1) includes all the data, whereas (B2) excludes one extremely high urinary adiponectin value and creatinine value that was obtained from patient No 33 with diabetes mellitus (DM). In (B1) $y=0.19 x+59.32, R^{2}=0.03$, and in (B2) $y=0.17 x+59.09$, $R^{2}=0.01$. (C) Correlation between urinary adiponectin level and ACR. (C1) includes all the data, whereas (C2) excludes one extremely high urinary adiponectin value that was obtained from patient No 33 with DM. In (C1) $y=46.30 x, R^{2}=0.85, p<0.01$ and in (C2) $y=30.86 x, R^{2}=0.49$. The relation between urinary adiponectin level and ACR shows a positive correlation. (D) Correlation between eGFR and ACR. (D1) includes all the data, whereas (D2) excludes one extremely high urinary adiponectin value that was obtained from patient No 33 with DM. In (D1) $y=-5.97 x+778.35, R^{2}=0.01$, and in (D2) $y=1.82 x+177.46, R^{2}=0.01$. ACR, albumin-to-creatinine ratio; eGFR, estimated glomerular filtration rate.

larger multimeric HMW (>300 kDa) form. ${ }^{27-30}$ There are two reports about the relationship between DM and adiponectin multimers in the blood. One report showed that mutations of human adiponectin related to DM are associated with impaired multimerization, consequent impaired secretion, and impaired adiponectin activity. ${ }^{29}$ The other report showed that an increase in the ratio of the HMW form to total adiponectin correlates with an 
A

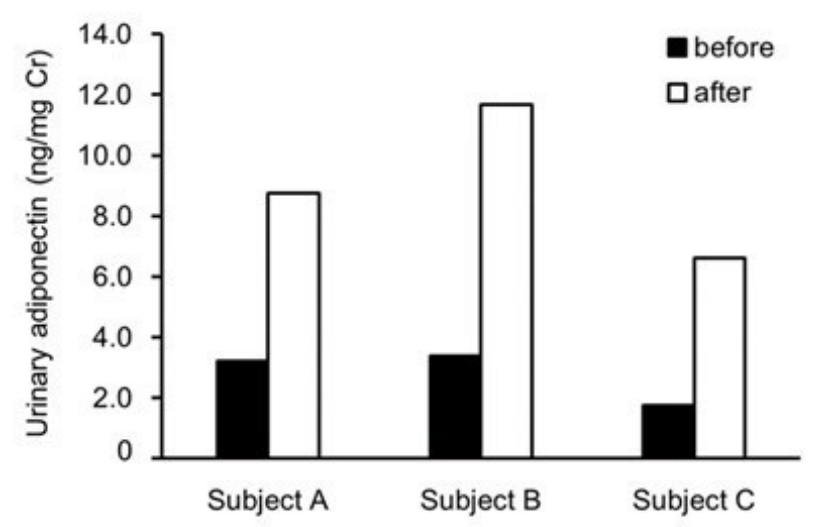

C

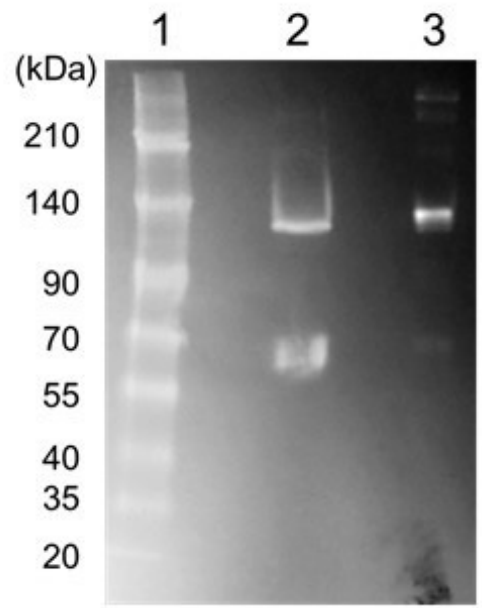

B

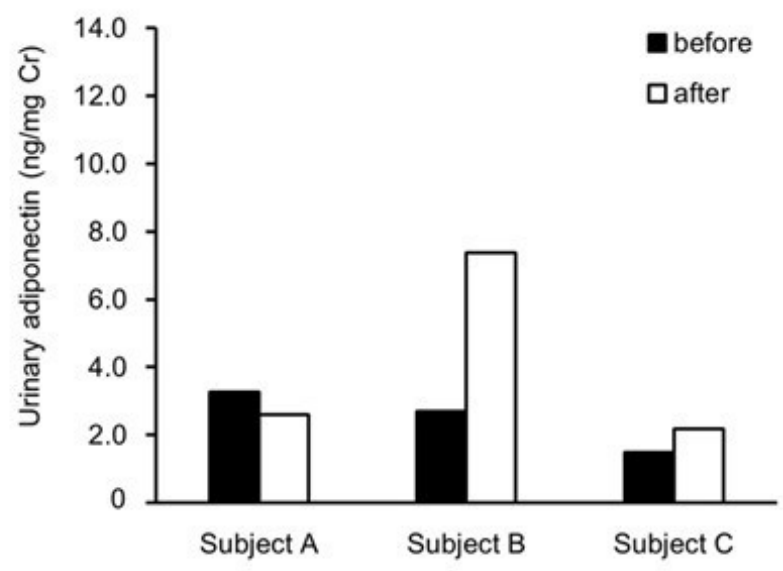

D

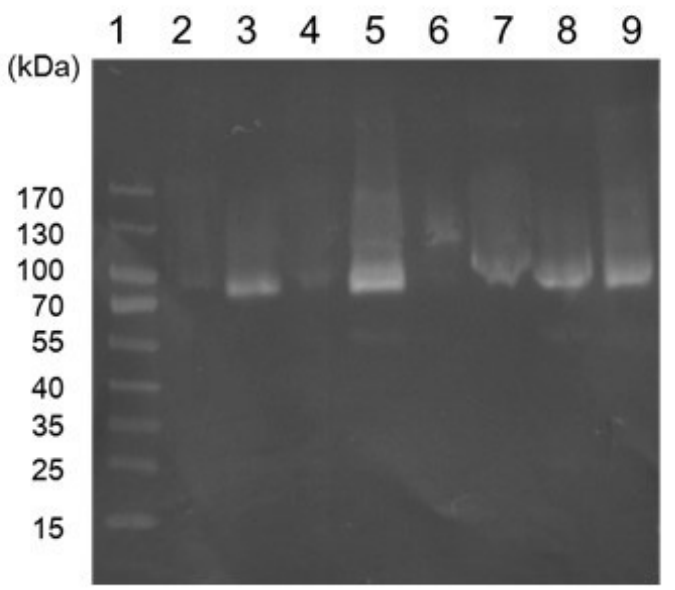

Figure 3 Exercise-induced changes in urinary adiponectin levels and difference in urinary adiponectin multimers between patients with diabetes mellitus (DM) and normal subjects. (A) Change induced by anaerobic exercise. (B) Change induced by aerobic exercise. Urine was collected before and after performing the exercise from three normal subjects, and their urinary adiponectin levels were determined. Anaerobic exercise evoked high urinary adiponectin levels (paired t-test, $p<0.05$ ), whereas there were no significant differences between before and after aerobic exercise (paired t-test, $p>0.05$ ). (C) Western blotting of urinary adiponectin in a patient (No 33) with DM. Lane 1: molecular weight markers; lane 2 shows the data for the antigen; and lane 3 contains the sample. In lane 2, low-molecular weight (LMW, a trimer) and medium-molecular weight (MMW, a hexamer) were detected, but not high-molecular weight (HMW, a large multimer). In lane 3, the main band comprised the MMW form, and the two weak bands comprised the LMW and HMW forms. (D) Western blotting of urinary adiponectin in the three normal subjects. Lane 1: molecular weight markers; lane 2: subject $A$ before anaerobic exercise; lane 3: subject $A$ after anaerobic exercise; lane 4: subject $B$ before anaerobic exercise; lane 5: subject $B$ after anaerobic exercise; lane 6 : subject $C$ before anaerobic exercise; lane 7: subject $C$ after anaerobic exercise; lane 8: subject $B$ before aerobic exercise; lane 9: subject $B$ after aerobic exercise. The bands were hardly observed before anaerobic exercise (lanes 2, 4, and 6), whereas the LMW band appeared after anaerobic exercise (lanes 3, 5, and 6). In (B), the urinary adiponectin level in one healthy subject (subject B) was changed by aerobic exercise. The urinary LMW adiponectin band was observed before (lane 8) and after (lane 9) aerobic exercise.

improvement in insulin sensitivity induced by a peroxisome proliferator-activated receptor- $\gamma$ agonist (thiazolidinedione) treatment. $^{31}$

We thus examined the adiponectin multimers in the urine to evaluate possible differences between patients with DM and normal subjects. When using urine of a patient with DM (No 33; see online supplementary table 1), we observed three adiponectin-related bands in western blotting (figure 3C). The main band was seen at MMW, which was a hexamer, and two weak bands were seen at LMW and HMW, which were a trimer and a large multimer, respectively (figure 3C). In the same western blotting data, we also visualized the LMW and MMW bands using an antigen that was used as the reference for the calibration curve for the ultrasensitive ELISA (figure 3C). Even though we could not observe a band for the HMW adiponectin in the antigen data, we confirmed that the ultrasensitive ELISA for urinary adiponectin measured the change in MMW during DM progression (figures 1 and 2).

In contrast, the bands were hardly observed in the western blotting data using the urine of normal subjects 
(figure 3D). As expected based on the ultrasensitive ELISA data for anaerobic exercise-induced changes in urinary adiponectin levels (figure 3A), LMW bands appeared in the normal subject urine samples after anaerobic exercise (figure 3D). Furthermore, the urinary adiponectin level in one normal subject also changed after aerobic exercise (figure 3B). We thus used western blotting to evaluate the urine sample of this normal subject and found that the urinary LMW adiponectin was present even before the aerobic exercise (figure 3D). Taken together, the LMW adiponectin sometimes appears in the urine of normal subjects, but if this adiponectin maintains the LMW form in urine, nephropathy is not a concern.

\section{CONCLUSIONS}

The findings of the present study demonstrated that urinary adiponectin could be a new diagnostic index for assessing CKD due to diabetic nephropathy. Progression of the CKD classification positively correlated with the urinary adiponectin level. Urinary adiponectin levels are more stable and reliable than ACR (ie, urinary albumin levels), which are used in the conventional CKD classification system. Our ultrasensitive ELISA coupled with thio-NAD cycling enables us to measure trace levels of urinary adiponectin in normal subjects. These results show that our ultrasensitive ELISA can be used to measure urinary adiponectin levels to distinguish between normal subjects and patients with DM and to estimate the progression of diabetic nephropathy. The higher urinary adiponectin levels in patients with DM are excreted as MMW adiponectin in urine. The MMW form is not detected in the urine of normal subjects; normal subjects excrete LMW adiponectin in their urine after anaerobic exercise.

These findings raise two questions. One is whether multimers of adiponectin can pass the glomerular filtration barrier (ie, leakage from glomeruli). In general, trace amounts of albumin $(66 \mathrm{kDa})$ and hemoglobin $(64 \mathrm{kDa})$ are filtered out, but larger molecular weight proteins are hardly filtered out. ${ }^{32}$ We thus consider that only LMW adiponectin $(84 \mathrm{kDa})$ can pass the glomeruli filter, and the MMW and HMW forms cannot. Our results support this notion because LMW adiponectin is excreted in normal subjects after anaerobic exercise (figure 3D). In other words, the urinary LMW adiponectin passes through the glomeruli filter at least in normal subjects. In contrast, the western blotting data showed that the LMW, MMW (168 kDa), and HMW (>300 kDa) forms were detected in the urine samples of patients with DM (figure 3C). At present, we do not know why MMW and HMW adiponectin appears in the urine of patients with DM. The filtration function of a glomerular basement membrane is controlled by different barrier types. One is a size barrier as described above, and the other is a charge barrier. ${ }^{33}$

As described earlier, thiazolidinediones (ie, peroxisome proliferator-activated receptor- $\gamma$ agonists) and sodium/glucose cotranspoter-2 (SGLT2) inhibitors are known to increase HMW adiponectin in serum. ${ }^{31}{ }^{34-36}$ In the present studies, we used thiazolidinediones and SGLT2 inhibitors to some of the patients with DM examined (online supplementary table 1). However, these drugs were not administered to the patient No 33 examined in figure 3C. That is, the multimers of urinary adiponectin were not caused by thiazolidinediones or SGLT2 inhibitors.

Another question is why adiponectin is a more reliable marker compared with albumin. Albumin is a 66 $\mathrm{kDa}$ protein that can easily pass the glomeruli filter with collapse of the filtration function or during physical exercise. For albuminuria (ie, measurement of albumin), a urine sample must be collected in an early morning, because it appears to be the most reliable for screening with high sensitivity and high specificity. ${ }^{37}$ However, our present results showed that the adiponectin measurement does not depend on the time of urine sampling. Further, to our knowledge, there are no multimer forms of albumin in urine. Therefore, an increased concentration of adiponectin multimers, but not of albumin monomers, reflects an increase in the CKD risk. Furthermore, urinary adiponectin levels seem stable (figure 2). Although the levels are stable, this does not suggest that the responsiveness of urinary adiponectin levels is poor, because we demonstrated a positive correlation between CKD classification and urinary adiponectin levels. We thus conclude that the emergence of MMW adiponectin in urine and increases in its levels are a good marker for diabetes nephropathy.

The potential cost of the present method would be suppressed at the same level as the conventional methods used for diagnosis of diabetes nephropathy. Instead of a microplate reader for measuring the absorbance of thio$\mathrm{NADH}$, we are now developing a dedicated apparatus for our ultrasensitive ELISA. In this apparatus, we use a disposable cartridge that includes the antibodies for a target protein and the reaction solutions. If the cut-off value of urinary adiponectin levels could be set at 4.0 $\mathrm{ng} / \mathrm{mg}$ creatinine, we would further suppress the cost. We think that our method can replace the conventional methods.

In conclusion, we developed an ultrasensitive ELISA, and measured urinary adiponectin levels in patients with DM and normal subjects. The urinary adiponectin levels in patients with DM were higher than normal subjects. This conclusion will be further confirmed by an increase in the number of subjects examined. The urinary adiponectin levels increased with an increase in the CKD risk. Urinary adiponectin formed MMW and HMW multimers in patients with DM, whereas it formed only an LMW multimer in normal subjects. Our new assay showed that urinary adiponectin could be a new diagnostic index for CKD.

Author affiliations

${ }^{1}$ Department of Biology, Waseda University, Shinjuku, Tokyo, Japan 
${ }^{2}$ R\&D Headquarters, TAUNS Laboratories, Izunokuni, Shizuoka, Japan

${ }^{3}$ Kagawa School of Pharmaceutical Sciences, Tokushima Bunri University, Sanuki, Kagawa, Japan

${ }^{4}$ Faculty of Medicine, Kagawa University, Miki, Kagawa, Japan

${ }^{5}$ Lipid Science and Aging Research Center, Kaohsiung Medical University, Kaohsiung, Taiwan

${ }^{6}$ Faculty of Pharmaceutical Sciences, Health Sciences University of Hokkaido, Ishikari-Tobetsu, Hokkaido, Japan

${ }^{7}$ Graduate School of Pharmaceutical Sciences, Hokkaido University, Sapporo, Hokkaido, Japan

${ }^{8}$ Graduate Institute of Medicine, Kaohsiung Medical University, Kaohsiung, Taiwan

Contributors KN, SW, KM, and El designed the study. SY, HC, MI, MM, YHJ, KS, HT, MK, AN, MN, HI, TA, TY, KM, JHC, SMC, YCS, MJZ, LYK, and CHC collected and analyzed the data. TY, TM, and El interpreted and analyzed the data. SY, HC, KM, TY TM and El wrote the manuscript. El is the guarantor of this work and, as such, had full access to all the data in the study and takes responsibility for the integrity of the data and the accuracy of the data analysis.

Funding This work was supported by a Grant for the Development of Systems and Technology for Advanced Measurement and Analysis from JST, a grant for the Regional Innovation Strategy Support Program from MEXT, Waseda University Grants for Specific Research Projects (2017A-015, 2019C-123) and the Precise Measurement Technology Promotion Foundation to El.

Competing interests MM, YHJ, KS, KN, and SW are employees of TAUNS Laboratories.

Patient consent for publication Not required.

Ethics approval The study was approved by the ethics committees at Waseda University, Tokushima Bunri University, and Kagawa University.

Provenance and peer review Not commissioned; externally peer reviewed.

Data availability statement All data relevant to the study are included in the article or uploaded as supplementary information.

Open access This is an open access article distributed in accordance with the Creative Commons Attribution Non Commercial (CC BY-NC 4.0) license, which permits others to distribute, remix, adapt, build upon this work non-commercially, and license their derivative works on different terms, provided the original work is properly cited, appropriate credit is given, any changes made indicated, and the use is non-commercial. See: http://creativecommons.org/licenses/by-nc/4.0/.

\section{REFERENCES}

1. Thorp ML, Eastman L, Smith $\mathrm{DH}$, et al. Managing the burden of chronic kidney disease. Dis Manag 2006;9:115-21.

2. Levey AS, Eckardt K-U, Tsukamoto Y, et al. Definition and classification of chronic kidney disease: a position statement from kidney disease: improving global outcomes (KDIGO). Kidney Int 2005;67:2089-100.

3. Webster AC, Nagler EV, Morton RL, et al. Chronic kidney disease. Lancet 2017;389:1238-52.

4. Levin A, Stevens PE. Summary of KDIGO 2012 CKD guideline: behind the scenes, need for guidance, and a framework for moving forward. Kidney Int 2014;85:49-61.

5. Taal MW. Chronic kidney disease: towards a risk-based approach. Clin Med 2016;16(Suppl 6):s117-20.

6. National Kidney Foundation. K/DOQI clinical practice guidelines for chronic kidney disease: evaluation, classification, and stratification. Am J Kidney Dis 2002;39(2 Suppl 1):S1-S266.

7. Matsuzawa Y, Funahashi T, Nakamura T. Molecular mechanism of metabolic syndrome $\mathrm{X}$ : contribution of adipocytokines adipocytederived bioactive substances. Ann N Y Acad Sci 1999;892:146-54.

8. - - - - - Shimomura I, Kihara S, et al. Importance of adipocytokines in obesity-related diseases. Horm Res 2003;60(Suppl 3):56-9.

9. Wolf G. Adiponectin: a regulator of energy homeostasis. Nutr Rev 2003:61:290-2.

10. Nishida M, Funahashi T, Shimomura I. Pathophysiological significance of adiponectin. Med Mol Morphol 2007;40:55-67.

11. Morikawa M, Naito R, Mita K, et al. Subattomole detection of adiponectin in urine by ultrasensitive ELISA coupled with thio-NAD cycling. Biophys Physicobiol 2015;12:79-86.

12. Sethna CB, Boone V, Kwok J, et al. Adiponectin in children and young adults with focal segmental glomerulosclerosis. Pediatr Nephrol 2015;30:1977-85.
13. Han SS, Bae E, Ahn SY, et al. Urinary adiponectin and albuminuria in non-diabetic hypertensive patients: an analysis of the especial trial. BMC Nephrol 2015;16.

14. von Scholten BJ, Reinhard H, Hansen TW, et al. Urinary biomarkers are associated with incident cardiovascular disease, all-cause mortality and deterioration of kidney function in type 2 diabetic patients with microalbuminuria. Diabetologia 2016;59:1549-57

15. Koshimura J, Fujita H, Narita T, et al. Urinary adiponectin excretion is increased in patients with overt diabetic nephropathy. Biochem Biophys Res Commun 2004;316:165-9.

16. Kopf S, Oikonomou $\mathrm{D}$, von Eynatten $\mathrm{M}$, et al. Urinary excretion of high molecular weight adiponectin is an independent predictor of decline of renal function in type 2 diabetes. Acta Diabetol 2014;51:479-89.

17. Panduru NM, Saraheimo M, Forsblom C, et al. Urinary adiponectin is an independent predictor of progression to end-stage renal disease in patients with type 1 diabetes and diabetic nephropathy. Diabetes Care 2015;38:883-90.

18. Ha KH, Kim DJ. Urinary adiponectin and progression of diabetic nephropathy in type 1 diabetes. J Diabetes Investig 2016;7:470-1.

19. Yamamoto M, Hujimoto $Y$, Hayashi S, et al. A study of high, middle and low molecular weight adiponectin in urine as a surrogate marker for early diabetic nephropathy using ultra-sensitive ICT-EIA. Ann Clin Biochem 2018;4563217748681.

20. Rovin $\mathrm{BH}$, Song $\mathrm{H}$, Hebert LA, et al. Plasma, urine, and renal expression of adiponectin in human systemic lupus erythematosus. Kidney Int 2005;68:1825-33.

21. Zha D, Wu X, Gao P. Adiponectin and its receptors in diabetic kidney disease: molecular mechanisms and clinical potential. Endocrinology 2017;158:2022-34.

22. Christou GA, Kiortsis DN. The role of adiponectin in renal physiology and development of albuminuria. J Endocrinol 2014;221:R49-R61.

23. Watabe $\mathrm{S}$, Kodama $\mathrm{H}$, Kaneda $\mathrm{M}$, et al. Ultrasensitive enzyme-linked immunosorbent assay (ELISA) of proteins by combination with the thio-NAD cycling method. Biophysics 2014;10:49-54.

24. Ito $\mathrm{E}$, Kaneda $\mathrm{M}$, Kodama $\mathrm{H}$, et al. Immunoreactive insulin in diabetes mellitus patient sera detected by ultrasensitive ELISA with thio-NAD cycling. Biotechniques 2015;59:361-7.

25. Nakatsuma A, Kaneda M, Kodama H, et al. Detection of HIV-1 p24 at attomole level by ultrasensitive ELISA with thio-NAD cycling. PLoS One 2015;10:e0131319.

26. Watabe S, Morikawa M, Kaneda M, et al. Ultrasensitive detection of proteins and sugars at single-cell level. Commun Integr Biol 2016;9:e1124201.

27. Nakano Y, Tobe T, Choi-Miura NH, et al. Isolation and characterization of GBP28, a novel gelatin-binding protein purified from human plasma. J Biochem 1996;120:803-12.

28. Pajvani UB, Du X, Combs TP, et al. Structure-function studies of the adipocyte-secreted hormone Acrp30/adiponectin. Implications FPR metabolic regulation and bioactivity. J Biol Chem 2003;278:9073-85.

29. Waki H, Yamauchi T, Kamon J, et al. Impaired multimerization of human adiponectin mutants associated with diabetes. Molecular structure and multimer formation of adiponectin. J Biol Chem 2003;278:40352-63.

30. Tsao T-S, Tomas E, Murrey HE, et al. Role of disulfide bonds in Acrp30/adiponectin structure and signaling specificity. Different oligomers activate different signal transduction pathways. J Biol Chem 2003;278:50810-7.

31. Pajvani UB, Hawkins M, Combs TP, et al. Complex distribution, not absolute amount of adiponectin, correlates with thiazolidinedionemediated improvement in insulin sensitivity. J Biol Chem 2004;279:12152-62

32. Tojo A, Kinugasa S. Mechanisms of glomerular albumin filtration and tubular reabsorption. Int J Nephrol 2012;2012:1-9.

33. Bohrer MP, Baylis C, Humes HD, et al. Permselectivity of the glomerular capillary wall. Facilitated filtration of circulating polycations. J Clin Invest 1978;61:72-8.

34. Phillips SA, Kung J, Ciaraldi TP, et al. Selective regulation of cellular and secreted multimeric adiponectin by antidiabetic therapies in humans. Am J Physiol Endocrinol Metab 2009;297:E767-E773.

35. Rose FJ, Webster J, Barry JB, et al. Synergistic effects of ascorbic acid and thiazolidinedione on secretion of high molecular weight adiponectin from human adipocytes. Diabetes Obes Metab 2010;12:1084-9

36. Kakuda H, Kobayashi J, Sakurai M, et al. Residual effect of sodium glucose cotransporter 2 inhibitor, tofogliflozin, on body weight after washout in Japanese men with type 2 diabetes. J Clin Med Res 2019:11:35-41.

37. Marshall SM. Screening for microalbuminuria: which measurement? Diabet Med 1991;8:706-11. 